\title{
Discourse Elements in English Academic Discourse
}

\author{
Yulia Chubarova \\ Ogarev Mordovia State University \\ Correspondence concerning this article should be addressed to Yulia Chubarova, Ogarev Mordovia State \\ University, Bolshevistskaya Str. 68, Saransk, Russian Federation, 430005. E-mail: chubarovaue@gmail.com
}

\author{
Natalia Rezepova \\ Plekhanov Russian University of Economics
}

\author{
Correspondence concerning this article should be addressed to Natalia Rezepova, Plekhanov Russian \\ University of Economics, Stremyanny lane, 36, Moscow, Russian Federation, 117997. \\ E-mail: natalia0309@list.ru
}

\begin{abstract}
This paper presents a study of discourse elements in spoken academic discourse - a lecture - and identifies their specificities. The study seeks to identify discourse elements in a wide body of research material; to study structural, functional and pragmatic features of discourse elements in terms of the implementation of the intentions of the speaker; to identify from the auditory analysis any prosodic features of discourse elements. Discourse elements are specifically defined from the point of view of their pragmatics: the intention of the speaker influences the language of the lecture and the way in which ideas are connected with words. The study on discourse elements included several stages. Research material consisted of audio recordings of Philology lectures to students studying English as a foreign language by three native speakers of English (General American standard of pronunciation), all of whom are professors at American colleges and universities. In total, 6 lectures were recorded; they formed $a$ wide body of research material lasting 7 hours 33 minutes. This wide body of research material consisted of 2306 linguistic facts, i.e. discourse elements in context. From these, 150 fragments containing various discourse elements were then chosen to form a narrow body of research material lasting 40 minutes. The phonetic research consisted of auditory analysis: dividing the fragments of discourses into syntagms; defining the boundaries of syntagms; specifying pitch movement, tone level and type of scale; using perceptual gradations of each prosodic feature, etc. Prosodic marking was carried out in accordance with the method of notation adopted at the Department of English Phonetics at Moscow State Teacher Training University (1997). Scaling enabled the classification and sorting of all the studied elements (discourse elements). The authors used the following types of scales: nominative, ordinal and interval. Structural analysis proved that discourse elements have different structure and may be one-word elements and predicative phrases S+P incorporated into the structure of the academic discourse. All discourse elements can be divided into two large groups - connecting elements (connectors) and pragmatic elements.

The results of the research show that the studied elements differ structurally and can perform various functions. The functions of the discourse elements, their structure, intentions of the speaker and also their position in the phrase determine their prosodic features
\end{abstract}

Keywords: discourse, institutional discourse, lecture, discourse elements, structure, function, frequency of use, prosodic features

Discourse as a text in a real communicative situation can be interpreted in different ways. It can be regarded as a «mental model» (Johnson-Laird, 2001), representing a generalized summary of our knowledge and understanding of the world; as a «frame model» (Minsky, 1969; Fillmore, 1976, 1982), arranging perception about various ways of behavior in typical situations; as a «script model» (Shank, 1975; Schank, Abelson, 1977), presuming development, shifts and adjustments of those typical situations. 
To study the communicative aspects of discourse, we use a «situational model» elaborated and offered by Teun Van Dijk (2008, 2009), which combines the principles of social categories analysis to define our understanding and behavior within a social context with personal subjective knowledge (evaluation, opinions, patterns), as well as a social-semiotic approach of Michael Halliday (2003) and his discourse model, interlocking empirical, logical, interpersonal and contextual levels of analysis.

In this work we have applied a sociolinguistic approach towards an understanding of discourse. This implies that discourse is communication between people belonging to a particular social group. According to Vladimir Karasik (2002), this statusoriented discourse can have an institutional character. There are many different kinds of institutional discourse in our community, such as scientific, mass media, political, religious, pedagogical, medical, military, etc.

Valeria Chernyavskaya (2006) highlights the social context of discourse in arguing that modern discourse analysis is focused on the character and level of influence of various extra-linguistic factors on the language of the discourse. Discourse analysis has to answer the question of how the author, the receiver of the message, the field of communication, the channel, the intention of the speaker and so determine discourse organization and its language.

\section{Materials and Methods}

\section{Definition of Academic Discourse}

By spoken academic discourse in this work we mean a kind of scientific and institutional discourse whose purpose is to inform students. For our research we have chosen the lecture as one of the basic monologic genres of scientific discourse: academic discourse is a homogeneous lecture devoted to one topic. The peculiarity of the studied academic discourse is that it has a special addressee that consists of students who study English as a foreign language, with the attendant difficulties caused by listening to a foreign language, which means listening to and understanding a foreign speech, implying a number of psychological challenges. One of them is the irreversibility of the process of perception of speech, which requires a great deal of attention from the listeners.

In addition, the lecturer needs to be able to navigate the communicative situation, feel the addressee, and anticipate their reaction in preparing lectures and directing educational interaction. This is particularly important, as the recipients are students who are learning English as a foreign language. Since it is not their native language the lecturer has to take into consideration the linguistic and cultural competence of the listeners, their ability to understand him/her. That is why the contact with the students and the need to create a free and easy atmosphere in the classroom are very important for the linguistic, communicative and pragmatic organization of the discourse.

Discourse must have a coherent text and explicit textual references. A substantial number of studies (Grosz, Sidner, 1986; Mann, Thompson, 1988; Martin, 1992; Foltz, Kintsch, \& Landauer, 1998; Marcu, 2000; Budayev, 2009) examine the principles of inner organization of the discourse and text. By discourse elements many linguists mean those elements that play a very important role in the organization and structure of the discourse. Anna Prokhorova (2007) points out that besides their cohesive functions they may also have some pragmatic aspects. Most discourse elements can express the opinion of the speaker, the relationship between the speaker and the listener, logical links between the ideas. They also can be studied from the point of view of pragmatics. The intention of the speaker can influence the language of the lecture and the way in which the ideas are connected with words.

English academic discourse has a wide range of discourse signals optimizing the process of its perception and aimed at the recipient. It should be noted that oral discourse, unlike the written one, makes the speaker facilitate perception of the material by various means of speech connectors, including discourse elements.

\section{Definition of Discourse Elements}

Connecting elements have different names in academic literature. In particular, Randolph Quirk (1955) names expressions, such as sort of, you see, you know, I mean, well, etc., as intimacy signals, which allow the listener to feel more at ease, to be closer to the interlocutor. There are also other terms for connecting elements of discourse, such as "linking signals", "fillers in", "discourse markers", "temporizers". There have been few attempts to describe and classify these elements and most have not been continued. As a result, there is not any well-established scientific term. The terminological inconsistency is caused by a multiplicity of approaches to the study of the connecting elements. Moreover, these terms are used to refer to a different set of units, which is determined by the specific objectives of the researcher.

Most linguists think that discourse connectors are the elements that play an important role in organizing the content and structure of the discourse in addition to their semantic and pragmatic aspects. Their pragmatic essence is to ensure the correct understanding of the 
discourse. Therefore, the most preferable term, in our opinion, is the term discourse elements proposed by Deborah Schiffrin (1994).

By discourse elements we mean segments of speech that connect at least two phrases. They also reflect the intention of the speaker and his/her reaction towards what he/she is saying. The research found that the group of these elements has, firstly, no fixed boundaries, and secondly, has elements that may be presented by different parts of speech. In our article the group of discourse elements is presented by adverbs (so, now, thus, therefore, anyway, finally, however, though, further, similarly, perhaps, maybe, probably, certainly, (un)fortunately, naturally, evidently, surely, obviously, actually, etc.), interjections (well, oh), numerals (first, second), performative verbs (I think, I'm sure, I suppose, I guess, I agree, I say, I hope, I expect, I mean, I assume, I believe, I remember, I forget, I recall, I daresay, etc.), set expressions (first of all, for example, on the one hand, on the other hand, in addition, at the same time, in other words, in terms of, in general, that sort of thing(s), of course, no doubt, as you know, if you remember, you know, as I said, as I mentioned before, etc.), syntactical constructions (Let's start with ..., Let's move on to ..., Do you have any questions?, etc.).

The lecture as a genre of academic discourse abounds in these discourse elements. Since a lecture may be quite long in time, it demands various elements in its connection, division and organization. This helps explain the great number of discourse elements in it. It is also the result of spontaneity, oral speech and improvisation.

\section{Research Material and Methodology}

The research material for our study was audio recordings of lectures to students who studied English as a foreign language, with lectures in Philology given by three native speakers of English (General American standard of pronunciation). Native speakers are professors at American colleges and universities. In total 6 lectures were recorded; they formed $a$ wide body of research material lasting 7 hours 33 minutes, consisting of 2306 linguistic facts, i.e. discourse elements in context. Later 150 fragments containing various discourse elements were chosen and formed a narrow body of research material lasting 40 minutes.

The research on the material was conducted in the following way. First, all the academic discourses - lectures - were recorded, then transposed into their written variants, and finally discourse elements were identified. The following aims of the analysis of academic discourse were formulated:

1. To identify discourse elements in the wide body of research material.

2. To study structural, functional and pragmatic features of discourse elements, in terms of the implementation of the intentions of the speaker.

3. To find during the auditory analysis prosodic features of discourse elements.

To meet these objectives, we used complex methods of research, which included: functional and semantic, formal and structural, pragmatic and auditory types of analyses, questioning of a native speaker, a statistical method for data analysis of linguistic material, scaling and linguistic interpretation of the results.

\section{Phonetic Research}

The phonetic research consisted of auditory analysis of all fragments of discourses. Recorded fragments were presented to two auditors. At the preparatory stage, the fragments were given to the auditor, a native speaker, without any special phonetic training but with a philological education. He was to listen to the material, fill in the questionnaire and do the following tasks:

1. Determine whether the recorded speech belongs to the standard of General American pronunciation or not.

2. Point out whether the speech belongs to an educated native speaker or not.

3. Specify what style the recorded speech is of: informational, academic, publicist, oratorical or conversational.

The native speaker easily answered the questions, with the results of the analysis as follows. The speech belonged to academic style, was of General American standard of pronunciation and belonging to an educated native speaker.

The second auditor was a Russian phonetician, fluent in English, phonetically trained and experienced in the analysis of the intonation of oral speech. The main objective of the auditor was the analysis of the intonation patterns of the selected fragments of discourses and determination of prosodic features of discourse elements. The analysis was conducted in terms of scales, terminal tones and pauses. The auditor was asked to:

1. divide the fragments of discourses to syntagms, define the boundaries of syntagms;

2. specify pitch movement, tone level and type of scale, focusing on prosodic features of particular discourse elements;

3. determine the duration and functional type of pauses, and tempo.

We used the following perceptual gradations of each prosodic feature:

1. Tone level: low, medium, high.

2. Terminal tones:

- Low Fall, Mid Fall, High Fall; 
- $\quad$ Low Rise, Mid Rise, High Rise;

- Fall-Rise;

- Rise-Fall;

- Rise-Fall-Rise;

- $\quad$ Low Level, Mid Level, High Level.

3. Duration of a pause: very short, short, average, long.

4. Type of a pause: syntactical, emphatic, hesitation, rhetorical.

5. Tempo of speech: slow, average, fast.

6. Loudness: decreased, normal, increased.

Prosodic marking was done in accordance with the method of notation adopted at the Department of English Phonetics at Moscow State Teacher Training University (1997).

\section{Results and Discussion}

\section{Scaling}

All discourse elements in the research were scaled, enabling us to classify and sort all the studied elements, which was very necessary given the huge amount of material. All quantitative data obtained was analyzed in accordance with the recommendations of Konstantin Belousov and Natalia Blaznova (2005). Scaling is a form of fixing some features of the studied objects by ordering them in a certain numerical system. All the elements have a certain point, or bar graph index, which reflects the position of the feature on the scale. The scales are based, for example, on the principle of increasing or decreasing the degree of manifestation of the observed feature. In our case, this feature is the frequency of use of various discourse elements. We used the following types of scales: nominative, ordinal and interval.

\section{Nominative Scaling}

With the help of nominative scale we classified discourse elements in terms of special features and principles they may have. The first feature is the function they may have. All the elements were classified according to the functions in the discourse. The second feature is their structural characteristic.

Structural analysis has demonstrated that discourse elements have different structure and may be oneword elements (so, now, thus, therefore, anyway, etc.) and predicative phrases $(\mathrm{S}+\mathrm{P})$ incorporated into the structure of the academic discourse (if you remember, as I mentioned before, do you have any questions?, etc.). Thus we found four groups of discourse elements: one-word elements, combinations of meaningful words with auxiliary words, phrases and incorporated phrases. This is shown in Table 1.
Table 1

Structure of discourse elements

\begin{tabular}{|c|c|c|c|}
\hline \multicolumn{4}{|c|}{ Discourse elements } \\
\hline $\begin{array}{l}\text { One-word } \\
\text { elements: } \\
\text { anyway, first, } \\
\text { second, next, } \\
\text { however, now, } \\
\text { then, well, } \\
\text { so, probably, } \\
\text { perhaps, etc. }\end{array}$ & $\begin{array}{l}\text { Combina- } \\
\text { tions of } \\
\text { meaningful } \\
\text { words with } \\
\text { auxiliary } \\
\text { words: } \\
\text { in general, in } \\
\text { other words, } \\
\text { in terms of, in } \\
\text { addition, in a } \\
\text { way, in fact, } \\
\text { of course, etc. }\end{array}$ & $\begin{array}{l}\text { Phrases: } \\
\text { I suppose, I } \\
\text { think, I say, } \\
\text { all right, I } \\
\text { would add, } \\
\text { I mean, the } \\
\text { idea is, I'm } \\
\text { sure. I have a } \\
\text { feeling, inter- } \\
\text { esting enough, } \\
\text { you know, etc. }\end{array}$ & $\begin{array}{l}\text { Incorporated } \\
\text { phrases: } \\
\text { do you fol- } \\
\text { low me?, do } \\
\text { you know } \\
\text { this term?, } \\
\text { have you any } \\
\text { tho ughts } \\
\text { about that?, } \\
\text { let's look at } \\
\text { the concrete } \\
\text { example, let's } \\
\text { move on to } \\
\text { the next tech- } \\
\text { nique, look at } \\
\text { the sentence, } \\
\text { you have } \\
\text { to be care- } \\
\text { ful here, etc. }\end{array}$ \\
\hline
\end{tabular}

We found that the structure of discourse elements is also of great importance so that in terms of structure we paid attention to the following features:

1. if discourse elements are used separately (they form a separate syntagm) or not used separately (they are the part of a syntagm) (see Fig. 1);

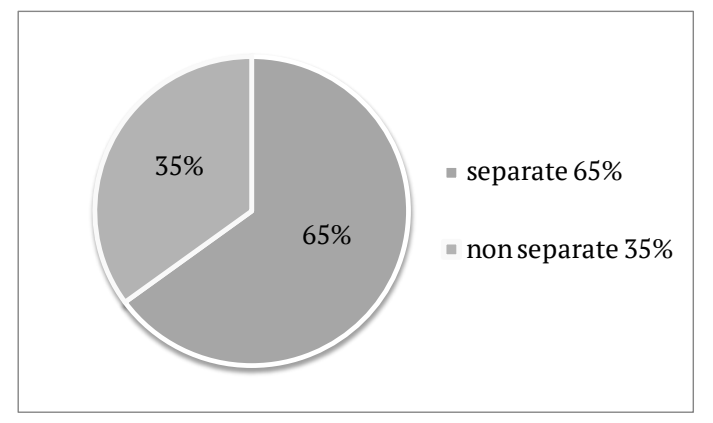

Figure 1. Separate/Non-separate discourse elements.

2. the position of a discourse element in a phrase (at the beginning, in the middle, at the end) (see Fig. 2).

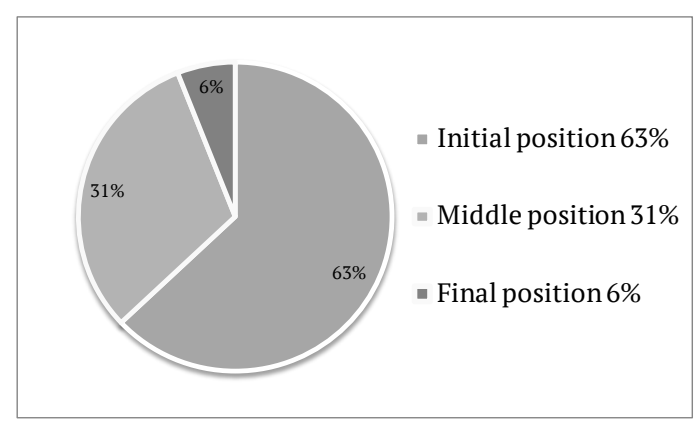

Figure 2. Position of a discourse element is a phrase. 


\section{Connectors and Pragmatic Elements}

Discourse elements have special peculiarities in spoken discourse. The lecturer tries to make discourse easier and more comprehensible for the students since the students can only listen to the speaker.

Discourse elements include not only elements that organize the discourse structurally but also elements that express the opinion of the speaker and are intended to establish some contact with the audience. That is why we have divided them into two big groups - connecting elements (connectors) and pragmatic elements (see Fig. 3).

The first group consists of elements that indicate linear connections in discourse, i.e. contextual connections. The second group consists of elements that realize the opposition lecturer-students and thus has elements that either express the opinion of the lecturer or are directed towards the audience and aim to create a collaborative setting between the speaker and the students.

As an example of discourse elements that function in academic discourse let us look at the following fragment:
If a writer writes poetry or screenplays as well as fiction that gives me a different idea what to expect than if a writer limits himself to one genre. You follow me? In other words, er some writers do write in all three genres, they write essays, they write poetry, they write fiction, they write scripts for dramas and for television programs. Er my feeling is often the more genres they write the perhaps the better qualified writers they are. Let me give an example, historically. Henry James is a famous $19^{\text {th }}$ century-early $20^{\text {th }}$ century American writer.

The group of pragmatic elements is presented by a direct question to the audience, You follow me? This element is very informal and shows the desire of the

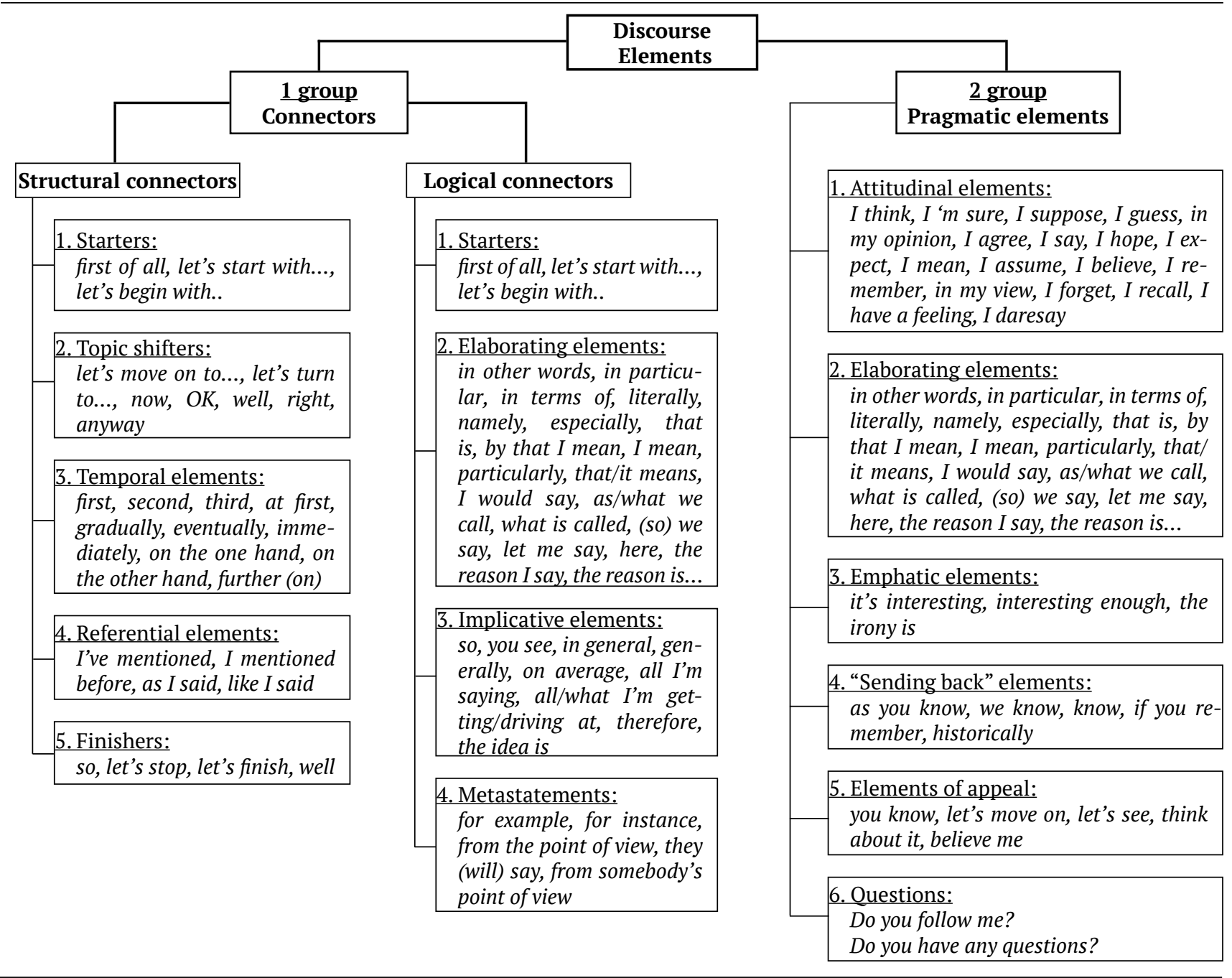

Figure 3. Discourse elements 
lecturer to get into contact with the audience and see how well they understand him. The discourse element, in other words, is one of the most frequently used and has the status of an elaborating element in this context. It belongs to the group of logical connectors. It is also necessary to note that the speech of the lecturer is very logical and consistent. With the help of discourse element, let me give you an example, historically, the lecturer wants to illustrate his opinion. This element also belongs to the group of logical connectors. The fact that the professor uses such elements shows that discourse has institutional character and that it is very important to him that his lecture is well understood by the students..

\section{Ordinal Scaling}

With the help of an ordinal scale discourse elements were classified as "more frequently used" and "less frequently used". In this scale it does matter in what order the cells in the scale are put. Using this particular scale we managed to arrive at conclusions about varieties of discourse elements and frequency of their use as shown in Table 2.

Table 2

Frequency of use of discourse elements

№ $\quad$ Discourse element $\quad \begin{gathered}\text { Frequency of } \\ \text { use of use of } \\ \text { the discourse } \\ \text { element (\%) }\end{gathered}$

1. So 15,3

2. Now

3. I think

4. Ok

5. In other words

6. In terms of

7. You know

8. For example

9. Well

10. I mean

11. (And) also

12. Anyway

13. Probably

14. That/it means

15. As/what we call

16. That sort of thing

17. I know

18. Yes/yeah

19. I/we would say

20. What/all I'm getting/driving at...
21. Maybe

22. At the same time 0,9

23. First of all 0,9

24. Do you follow me? $\quad 0,8$

25. It is/was interesting $\quad 0,8$

26. No 0,7

27. Of course 0,7

28. Any questions? / Do you have any 0.7 questions? / Anybody ask questions about ...? /Are there any questions you might have? / Let me ask if any questions you had about ...?

29. Right 0,6

30. Particularly 0,5

31. Let's turn to... / Let's look at... / Let $\quad 0,5$ me get to ... / Let's do smth. / Let's try smth.

32. In fact 0,4

33. I hope 0,4

34. I don't know $\quad 0,4$

35. Actually 0,4

36. I'm not sure $\quad 0,4$

37. Again 0,4

38. Primarily 0,3

39. Generally 0,3

40. Usually 0,3

41. And so forth 0,3

42. Fortunately/unfortunately 0,3

43. Apparently 0,3

44. (As) I understand $\quad 0,3$

45. In a way 0,3

46. (As) I say 0,3

47. Have you heard that term/word? / Do 0.3 you know that term? / Do you know what the word ... means? / Is that term you heard/know? / Is that the word you're clear on?

48. I'll give you the example. / Let me give $\quad 0,3$ you an example.

49. Have you heard of ... ? 0,3

50. (So) we say 0,2

51. I'm sure 0,2

52. That doesn't mean $\quad 0,2$

53. I guess 0,2

54. By that I mean $\quad 0,2$

55. What is called 0,2

56. I believe 0,2

57. I will say 0,2

58. On the other hand $\quad 0,2$ 


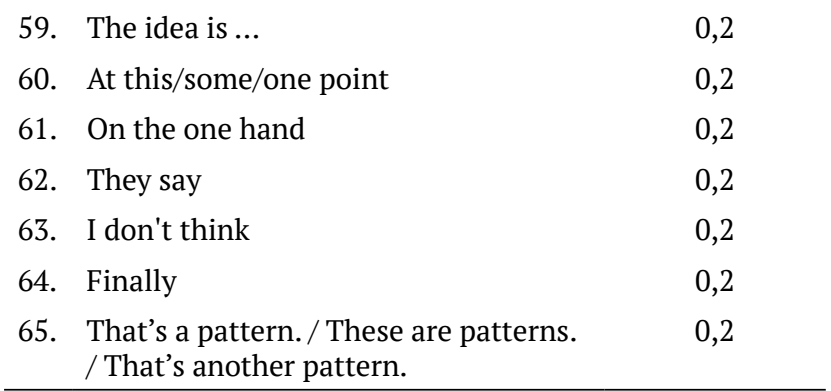

We also conducted statistic analysis of language material in terms of average statistic figures (discourse elements). As the result of this analysis we found the most frequently used discourse elements are: so, now, I think, OK, in other words, in terms of, you know, for example, well, I mean, (and) also, anyway, probably, that/it means. The results of this analysis allow us to conclude that there are a special number of discourse elements that are regularly used in this kind of discourse and that they vary in their functions. However the most commonly used are the elements that express result or consequence; shift the topic; clarify the opinion of the speaker (so, now, I think, OK, in other words, in terms of, you know, for example, well, I mean, (and) also, anyway, probably, that/it means). The use of such discourse elements can be explained by the desire of the lecturer to make students understand his ideas and concepts. This pragmatic goal is essential for the professor especially taking into consideration he/she is delivering a lecture to non-native speakers of English.

\section{Interval Scaling}

Interval scales enabled us not only to classify discourse elements but also to numerically express and compare them. Thus we received the data about the number of discourse elements and predominance of their prosodic characteristics. The analysis and interpretation of the data collected proved our hypothesis that the prosodic variations of discourse elements are determined by the intention of the speaker, the complexity of the structure of the elements, their position in the sentence and in the phrase (Table 3).

Table 3

Position of a discourse element in the phrase

\begin{tabular}{llccc}
\hline \multirow{2}{*}{ No } & \multirow{2}{*}{ Discourse element } & \multicolumn{3}{c}{ Position (\%) } \\
\cline { 3 - 5 } & & Initial & Middle & Final \\
\hline 1. & So & $\mathbf{9 8}$ & 2 & - \\
2. & Now & $\mathbf{9 8}$ & 2 & - \\
3. & I think & 46 & $\mathbf{5 1}$ & 3 \\
4. & In other words & $\mathbf{1 0 0}$ & - & - \\
5. & In terms of & 16 & $\mathbf{8 3}$ & 2
\end{tabular}

6. You know

7. For example

8. Well

9. I mean

10. (And) also

11. Anyway

12. Probably

13. That/it means

14. As/what we call

15. That sort of thing

16. I know

17. Yes/yeah

18. I/we would say

19. What/all I'm getting/driving at...

20. Maybe

21. At the same time

22. First of all

23. It is/was interesting

24. No

25. Of course

26. Right

27. Particularly

28. In fact

29. I hope

30. I don't know

31. Actually

32. I'm not sure

33. Again

34. Primarily

35. Generally

36. Usually

37. Say

38. And so forth

39. Fortunately/unfortunately

40. Apparently

41. (As) I understand

42. In a way

43. (As) I say

44. (So) we say

45. I'm sure

46. That doesn't mean

47. I guess

48. By that I mean

49. What is called

50. I believe 


\begin{tabular}{llccc} 
51. I will say & $\mathbf{8 0}$ & 20 & - \\
52. & On the other hand & $\mathbf{1 0 0}$ & - & - \\
53. & The idea is ... & $\mathbf{7 5}$ & 25 & - \\
54. & At this/some/one point & $\mathbf{5 0}$ & 25 & 25 \\
55. & On the one hand & $\mathbf{1 0 0}$ & - & - \\
56. & They say & - & 80 & 20 \\
57. I don't think & $\mathbf{7 5}$ & 25 & - \\
58. & Finally & - & $\mathbf{1 0 0}$ & - \\
\hline
\end{tabular}

The quantitative study showed that discourse elements are often used at the beginning of the phrase (63\%). They include discourse elements such as: so, now, in other words, well, anyway, yes/yeah, what/all I'm getting/driving at, I mean, (and) also, that/it means, I know. The lecturer uses such elements in the initial position to make contact with the students, to attract some attention to particular ideas and also to use this time to figure out what to say next. Such elements normally form a separate syntagm (69\%); the terminal tone is Medium Level; tempo and loudness are average. For example,

\footnotetext{
Becauses owhat what 'colleges have 'found outsis $\{$ they can>tel| historically from

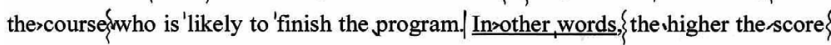
- the-more 'likely the 'student has a'bility to 'handle the university or 'college 'level work. And sso, they-tend'not to'take,students\} who have low , scores.
}

$31 \%$ of discourse elements are used in the middle of the phrase. The most often used elements in this position are: I think, in terms of, you know, for example, probably, as/what we say, I/we would say, maybe. Used in this position they may be ordinary remarks or additions to the main phrase. Or they can be fillers of pauses of hesitation. The speaker casually expresses his/her opinion towards the mentioned facts. He/she may use such elements to make the lecture livelier and let it sound more natural. All these factors determine prosodic realization of the elements. The intonation is normally the same as the one in the preceding syntagm. Very often these elements do not form a separate syntagm (I think, in terms of, probably, maybe). For example,

Ivlived in 'London for a year. And I can־still go'back to London\}and nnot repeat my,selß insterms of places I visited.

Sometimes discourse elements in this position can form a separate intonation group (44\%), separated with pauses and realized with a particular terminal tone. Very often it is Medium Level $(44,4 \%)$ and LowRise $(39,8 \%)$. The tempo and loudness are average. For example,

\footnotetext{
And there're people who have llonger vacations. $\rightarrow$ Namely teachers $\{\operatorname{er}\{$ for examplefwon't be,teaching in thermonths of July and.August\{most of the, time.|
}

Discourse elements in the final position appear quite seldomly (6\%). They are normally used here to attract the attention of the listeners and emphasize the idea mentioned. In this position you can find elements such as: sort/type of thing, and so forth, I don't know, I'm not sure. They usually form a separate intonation group (92\%) with Low/Medium-Fall (45, 5\%) and Medium Level (20\%) terminal tones; average tempo and loudness. For example,

\footnotetext{
So altogether $\{$ an American employeesstarting_out\{can expect about fifteen to sixteen 'paid holidays $\{$ vacation, time. $>$ Now that may,sound like svery 'little by Russian, standards, $\{$ I'minot,sure.
}

The analysis of the prosodic characteristics of the incorporated phrases lets us say that these elements are usually marked by high, increasing loudness and slow tempo. The terminal tone depends on their structure and the emotional level of the lecturer. Sometimes they are marked by rhetorical pauses. It all allows us to mark them out in the context and also optimize the interpretation of the lecture. For example,

\footnotetext{
That may be'only a collection of imageşjust a collection of images $\{$ that are ¿somehow related. OK, |ergany-questions so far?

$\rightarrow$ Also I-think these are'main, thing Iswanted to cover. |Now, do you 'have

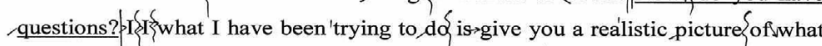
'life for 'young' college' graduate is $S_{\text {in the United_States. }}$
}

\section{Conclusion}

The process of interaction between the lecturer and the students and its result are considered in this research as a kind of institutional discourse, particularly academic discourse. There are many discourse elements in this kind of discourse that make it institutional. They also provide cohesion and realize one of the principles of lectures, that is, a focus on the audience; in our case it is a student audience that studies English as a foreign language. The specific addressee determines special choice of discourse elements and their frequency of use.

The studied elements differ structurally and can perform different functions. The most common function is delimitation and at the same time integration of the fragments of the discourse. Another function can be connected with expressing the personal opinion of the lecture and his/her judgements. That is why we have divided discourse elements in our research into two big groups that are subdivided into smaller groups.

The functions of the discourse elements, their structure, intentions of the speaker and also their position in the phase determine their prosodic features. They are normally marked out in the discourse and thus help the students to comprehend the lecture 
better. These findings contribute to our understanding of discourse elements representation and their functioning in academic discourse considering specific features of student audience. The obtained results open perspectives for further research on this issue. Interesting and promising, in our opinion, seems a further study of discourse elements, their functional characteristics and prosodic features on the material of different kinds of discourse, as well as different varieties of English.

\section{References}

Belousov, K. I., \& Blaznova, N. A. (2005). Introduction to experimental linguistics. Moscow, Russia: Flinta, Nauka.

Budayev, E. V. (2009). The missing link in discourseanalysis: T. van Dijk's contextual models. Political Linguistics, 28, 153-155.

Chernyavskaya, V. E. (2006). Discourse of power and power of discourse: Problems of speech influence. Moscow, Russia: Flinta.

Fillmore, C. J. (1976). Frame semantics and the nature of language. In Annals of the New York Academy of Sciences: Conference on the Origin and Development of Language and Speech (Vol. 280, pp. 20-32). New York, NY: The New York Academy of Sciences.

Fillmore, C. J. (1982). Frame semantics. In Linguistics in the Morning Calm (pp. 111-137). Seoul, South Korea: Hanshin Publishing Co.

Foltz, P. W., Kintsch, W., \& Landauer, T. (1998). The measurement of textual coherence with latent semantic analysis. Discourse Processes, 25(2-3), 285-307.

Grosz, B., \& Sidner, C. (1986). Attention, intention, and the structure of discourse. Computational Linguistics, 12(3), 175-204.

Halliday, M. A. K. (2003). On language and linguistics. London, UK: Continuum International Publishing.

Johnson-Laird, P. N. (2001). Mental models and deduction. Trends in Cognitive Science, 5, 434-442.

Karasik, V. I. (2002). Language circle: Personality, concepts, discourse. Volgograd, Russia: Peremena.

Mann, W. C., \& Thompson, S. A. (1988). Rhetorical structure theory: Toward a functional theory of text organization. Interdisciplinary Journal for the Study of Discourse, 8(3), 243-281.

Marcu, D. (2000). The theory and practice of discourse parsing and summarization. Cambridge, MA: MIT Press.

Martin, J. (1992). English text. System and structure. Amsterdam, Netherlands: John Benjamin Publishers.

Minsky, M. (1969). Semantic information processing. Cambridge, MA: MIT Press.

Prokhorova, A. A. (2007). Prosodicheskoe oformlenie diskursivnyh svjazej $v$ ustnom monologicheskom tekste (teoretiko-jeksperimental'noe issledovanie) [Prosodic features of discourse connectors in spoken monologic text (theoretical and experimental research)] (Unpublished doctoral dissertation), Yaroslavl State Pedagogical University, Yaroslavl, Russia.

Quirk, R. (1955). Studies in communication. London, UK: Pitman.

Shank, R. C. (1975). SAM (Script Applier Mechanism) A story understander. Research Report, 43, 151-157.

Schank, R. C., \& Abelson R. P. (1977). Scripts, plans, goals, and understanding: An inquiry into human knowledge structures. Hillsdale, NJ: Lawrence Erlbaum Associates.

Schiffrin, D. (1994). Approaches to discourse. Oxford, UK: Oxford University Press.

Sokolova, M. A. (1997). Prakticheskaja fonetika anglijskogo jazyka [Practical phonetics of the English language]. Moscow, Russia: Vlados.

van Dijk, T. (2008). Discourse and context: A sociocognitive approach. New York, NY: Cambridge University Press.

van Dijk, T. (2009). Society and discourse: How social contexts influence text and talk. New York, NY: Cambridge University Press. 